1.Sarah H. Norgate, ${ }^{\mathrm{a}}$ Nigel Davies, ${ }^{\mathrm{b}}$ Chris Speed, ${ }^{\mathrm{c}}$ Tom Cherrett ${ }^{\mathrm{d}}$ and Janet Dickinson ${ }^{\mathrm{e}}$

2. Word counts:

Abstract: $=54$
Main text: $=1000$
References: $=205$

Entire text (total + addresses): $=1393$

3. The Missing Dimension: The Relevance of People's Conception of Time

4. Sarah H. Norgate, ${ }^{\mathrm{a}}$ Nigel Davies, ${ }^{\mathrm{b}}$ Chris Speed, ${ }^{\mathrm{c}}$ Tom Cherrett, ${ }^{\mathrm{d}}$ and Janet Dickinson ${ }^{\mathrm{e}}$

5. ${ }^{a}$ University of Salford, ${ }^{b}$ Lancaster University, ${ }^{\mathrm{c}}$ The University of Edinburgh; ${ }^{\mathrm{d}}$ University of Southampton; ${ }^{\mathrm{e}}$ Bournemouth University.

6. ${ }^{a}$ Psychology and Public Health, University of Salford, Greater Manchester, M6 6PU, United Kingdom; ${ }^{\mathrm{b}}$ School of Computing \& Communications, Lancaster University, LA1 4YR, United Kingdom; ${ }^{c}$ Edinburgh College of Art, The University of Edinburgh, EH3 9DF; United Kingdom; ${ }^{\mathrm{d}}$ Transportation Research Group, University of Southampton, S017 1BJ,

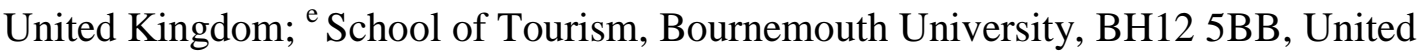
Kingdom.

7. Sarah Norgate +44 161 2952324; Tom Cherrett +44 2380594657

8. S.H.Norgate@salford.ac.uk nigel@comp.lancs.ac.uk c.speed@ed.ac.uk T.J.Cherrett@soton.ac.uk JDickinson@bournemouth.ac.uk

9. http://www.seek.salford.ac.uk:80/pp.jsp?NorgateSarah1374

\title{
10. Abstract
}

While a timely conceptual innovation for the digital age, the "map" proposed by Bentley et al. would benefit from strengthening through the inclusion of a perspective from non clock time perspective. In this way, there could be new hypotheses coined which could be applied and tested relevant to more diverse societies, cultures and individuals. 


\section{Maintext}

Central to the authors" vision is a "map" that captures the essence of human decision making around collective behaviour in the digital age. Key to their argument, is the expectation that decision making is shifting towards imitation or herding driven by popularity and without grounding in the knowledge of the benefits of the behaviour. In explanation for this phenomenon, the authors focus on economies replete with online communication where people inhabit an information ecology characterised by data overload in terms of both the number of sources of intelligence and the number of choices on offer.

We argue that the authors' characterisation of decision making occurring under conditions of time constraint this biases the "map" towards "clock time" culture. We suggest that adopting the "map" as an empirical framework appropriate for hypothesis testing may be premature unless the signature of the behavioural pattern also takes into account temporal perspectives anchored by alternative established societal and/or individual relationships with time. Specifically, the alternatives that we consider include a focus on 'event' time, together with a broadening of orientation towards future time.

The distinction between clock time and event time has been made socio-historically, particularly in relation to the rise of clock time culture associated with mass timetabling consequent on introduction of the first passenger train in England in the 1820s (Zerubavel, 1982) and the subsequent industrial revolution in Western Europe. More recent distinctions between clock and event cultures focus on different behaviour patterns associated with each tendency. For instance, in clock time, activities are typically completed one at a time in sequence. In contrast, in 'event time', the inclination is to shift 'to and fro' across tasks 
without any clock-determined schedules. One notion is that each society displays an overriding 'average' set of behavioural markers towards either clock time or event time whilst individuals within the society/culture will also display their own tendencies (e.g. Hall, 1959) which correspond with these societal norms to a lesser or greater degree. In this respect, whilst the USA and Western Europe are considered to focus on 'clock-time', South or Central America, Middle East, and South Europe are more event focused (Lindquist \& Kaufman-Scarborough, 2007).

Although there is a risk of oversimplification in adopting any conceptual dichotomy, the narrative around differentiation between the notions of 'clock' time and 'event' time (Levine, 1997) has been theorized around the concepts of 'monochronicity' and 'polychronicity' (Hall, 1959), particularly in relation to workplace behaviour. 'Monochronicity' refers to the tendency to colonize time with one activity at a time whilst 'polychronicity' revolves around doing more than one activity simultaneously.

Treatment of time as an economic resource is a hallmark of clock time where decision-making takes place under time constraints. However, in the big data era, clock time is not necessarily exclusively dominant in economies replete with online communication. For instance, take Japan. With a pervasive online economy and around 80 per cent of the population accessing the internet (MMG, 2013), yet the culture functions on a combination of 'clock' and 'event' time (Tsuji, 2006). In addition, in rural India, there is a growing reliance on the mobile internet, against a backdrop where the predominant mode is 'event' time.

Further, recent evidence suggests that there are discernible differences in the way humans self-regulate (Avnet \& Sellier, 2011). People with a 'self-regulation' couched in an approach known as 'prevention' engage in activity which reduces the number of errors until no further risks are discernible. In this way, they are internally guided, so aligning with an event time rather than an external temporal cue. In contrast, individuals characterised with a 
self-regulation approach known as 'promotion' rely less on internal resources to determine when a job is done and instead prioritise external cues such as time cues to ensure a timely completion of tasks.

Evidence from across social science converges to suggest alternatives exist to clock time. Within the digital age, this has implications for decision making processes. In terms of trends which may guard against a shift to the southeast or provide an alternative conceptualisation of it, studies of cultural differences in decision making reveal a number of findings which suggest that the tendency towards herd-like outcomes may be less likely once we take a temporal cultural perspective.

A number of studies reveal cultural dimensions to decision making. For instance, in a dynamic simulation task called COLDSTORE, individuals in clock time cultures (e.g. USA, Germany) tend towards "adaptor-type" decision making compared with those from event cultures (e.g. Indian, Filipino and Brazil) who engage in more "oscillator-decision" making characterised by taking the present situation into account (Güss \& Dörner, 2011). Significantly, whilst the "map" proposed by Bentley et al. focused on the role of the 'past' and the 'present', adopting a cultural perspective would mean taking into account the work by Zimbardo and Boyd (1999) where people have a predominant disposition to a 'future' time orientation. The "map" could be strengthened by reflecting those cultures which display a time orientation towards the future, which may mean that the authors have underestimated of the extent to which those populating below the 'equator' in the South East quadrant of the "map" can perceive benefits. We know, for instance, from work on risk-taking that cultures with more hierarchial views underestimate risk because they defer to the expertise of experts when making choices (Dake, 1991).

Further of relevance to the mechanism of 'imitation' associated with "herding", increased imitation can be desirable. For instance, in sustainable transport, making real time 
traffic jam on highways visible to prospective commuters via public display systems, has the scope to discourage imitation of a 'popular' trip mode taken by previous commuters. On a project called Sixth Sense Transport we are eliciting imitation of pro-environmental behaviour through design of Apps designed to achieve this.

In conclusion, we would like to persuade the authors to extend their "map" with a cultural perspective that reflects alternative conceptions of human relationships with time, and would enable social scientists to coin new testable hypotheses in topical domains within society.

12.

Avnet, T., \& Sellier, A.-L. (2011). Clock time vs. event time: Temporal culture or selfregulation? Journal of Experimental Social Psychology, 47, 665-667. doi: 10.1016/j.jesp.2011.01.006

Dake, K. (1991) Orienting dispositions in the perception of risk: An analysis of contemporary worldviews and cultural biases. Journal of Cross-Cultural Psychology, 22, 61-82.

Güss, C.D. \& Dörner, D. (2011). Cultural differences in dynamic decision-making strategies in a non-linear, time-delayed task. Cognitive Systems Research, 12, 365-376. doi: 10.1016/j.cogsys.2010.12.003

Hall, E.T. (1959). The Silent Language. Garden City, New York: Doubleday. Levine, R. (1997). A geography of time: The temporal misadventures of a social psychologist. Or how every culture keeps time just a little bit differently. New York: Basic Books.

Lindquist, J.D., \& Kaufman-Scarborough, C.J. (2007). The Polychronic-Monochronic Tendency Model: PMTS scale development and validation. Time and Society, 16(2/3), 269-301. doi: 10.1177/0961463X07080270 
MMG (Miniwatts Marketing Group) (2013). Asia Internet Use, Population Data and Facebook Statistics [Online] Available from:

http://www.internetworldstats.com/stats.htm [Accessed 29th April 2013]

Sixth Sense Transport (2013) Available from: http://www.sixthsensetransport.com/[Accessed 29th April 2013]

Tsuji, Y. (2006). Railway Time and Rubber Time: The paradox in the Japanese conception of time. Time and Society. 15, 2/3, 177-195. doi: 10.1177/0961463X06066950

Zerubavel, E. (1982). The standardization of time: A sociohistorical perspective. The American Journal of Sociology, 88(1), 1-23. 